\title{
Ideas for Using Fitbits to Assess Physical Activity in Children
}

\author{
Kristen Welker ${ }^{1}$, Jalisa Elkins ${ }^{2}$ and Laura Nabors ${ }^{1 *}$ \\ ${ }^{1}$ Health Promotion and Education Program, School of Human Services, University of Cincinnati, USA \\ ${ }^{2}$ Cincinnati Recreation Commission, Oakley Recreation Center, USA
}

\section{Introduction}

A sedentary lifestyle is related to childhood obesity and can be improved through behavior change [1]. School-based obesity prevention programs can be a way to teach children how to maintain a healthy lifestyle, which includes increasing activity levels [2]. We have worked to incorporate obesity prevention programming in after-school programs (e.g.: [2-4]). Lessons feature information on healthy eating and improving levels of physical activity at home. Our research has shown positive impact through parent and child report of positive change in healthy eating and increased exercise levels $[2,3]$. We have been working to collect data on children's physical activity levels using Fitbits.

\section{Challenges Using Fitbits with Children}

We have encountered several challenges in using Fitbits. For instance, participant attendance was inconsistent, so we could not distribute Fitbits as we had intended. In addition, children did not always wear the Fitbits consistently and for the entire day. At first, the children were excited to wear the Fitbits and were eagerly hoping to be selected to wear one for the week. The children enjoyed seeing the lights increase each day as they took more steps. Unfortunately, some of the children were so interested in checking their lights it became a distraction during class, and teachers requested the children not wear them at school. Other children took them off at home at the request of their parent, to play, or because they felt they were too big or cumbersome to wear. Most of the children took their Fitbits off for a few hours, which led to inaccurate measurement of their daily activity. As time went on, the novelty of the Fitbit had worn off and it was a challenge to find enough interested students to wear a Fitbit. There were several instances when a member of our team went to collect a Fitbit only to find it in a backpack or at home. Several of the children lost their Fitbits.

\section{Ideas for Overcoming Challenges}

Those who are interested in implementing Fitbits into childhood obesity prevention programs, may learn from the issues we faced, which are presented in Table 1.

Our team believes a more colorful or fun-looking step counter or accelerometer may be helpful in maintaining child interest. We used plain black or blue bands. There are Fitbits and trackers which have bands in many colors and others with fun designs or characters on the bands. Providing children with incentives for wearing the Fitbits, such as prizes for keeping them on and for returning them, might have improved data collection. We also were unable to use the full functionality of the Fitbit app and had to use it count steps per day. In order to use more options on the Fitbit app, we would recommend using a Fitbit with a single piece design, as opposed to the two-piece design of the Fitbit Flex 2. Using a reward system, with daily rewards for wearing the Fitbits might have encouraged children to wear them more often. Talking to teachers, especially physical education teachers, so that they understood reasons for children wearing the Fitbits may have provided a rationale for children to be able to wear them at school.

With respect to data collection for those working in recreation and school settings, we recommend using a simplified design. We tried to collect data on three days of the week, each week, over an eight-week program. Attempting to gather data each week seemed to overwhelm the children. Using a pre- and post-intervention design, collecting activity data before and after an intervention, may yield more accurate data. It may be necessary to include incentives for the child to perform better each week, or create some sort of step count competition amongst the program participants if data are collected on a weekly basis. Another idea to involve children in assessment would be to have ceremonies with the group to share step results. This might assist in holding children's interest in tracking their activity and may even encourage them to engage in additional activity as an intervention is delivered.

\section{Conclusion}

Fitbits can be an incredibly valuable tool in measuring and encouraging physical activity in children. However,

*Corresponding author: Laura Nabors, Health Promotion and Education, School of Human Services, University of Cincinnati, Cincinnati, Ohio, USA, Tel: 45221-0068

Accepted: March 23, 2019

Published online: March 25, 2019

Citation: Welker K, Elkins J, Nabors L (2019) Ideas for Using Fitbits to Assess Physical Activity in Children. J Healthcare 2(1):14-15 
Table 1: Difficulties Using Fitbits and Ideas for Success.

\begin{tabular}{|l|l|}
\hline Issues Encountered & Possible Solution(s) \\
\hline Inconsistent use & Reward students in some way for wearing Fitbit consistently. \\
\hline & Work with the students' teachers to receive help with monitoring use during class. \\
\hline & Provide incentives for the parent/family for the child's consistent participation. \\
\hline Develop a step contest to encourage activity while wearing the band. \\
\hline $\begin{array}{ll}\text { Hold a "closing ceremony" type of celebration to finalize the study where students and } \\
\text { families can learn the final step count results and other study information. }\end{array}$ \\
\hline Limit the amount of time spent wearing the Fitbit. \\
\hline Let the students know how they are doing: Allow them to watch the process of checking \\
\hline their steps online/on a phone.
\end{tabular}

it is important to note that Fitbits are a tool, among many measurement tools, to help those working with children in community settings. Fitbits can be useful to assess light activity, but may not be the best tool for assessing moderate and high levels of activity [5]. We found that implementing Fitbits for data collection was challenging and it was costly when Fitbits were lost or not returned. We hope that in sharing our struggles, those seeking to assess change in children's activity levels using Fitbits can learn from our issues and see success in their efforts. Moreover, we believe that encouraging measurement of community-based programs is important, and more finding for evaluation of these programs will allow evaluators to use direct and indirect or proxy methods, such as Fitbits, to assess child involvement in physical activity. Given that obesity is such a pervasive national problem [1], continuing to find ways to assess child involvement in physical activity will provide critical information for those designing and implementing programs to help prevent obesity in children.

\section{References}

1. Katzmarzyk PT, Barreira TV, Broyles ST, et al. (2015) Relationship between lifestyle behaviors and obesity in children ages 9-11: Results from a 12-country study. Obesity (Silver Spring) 23: 1696-1702.

2. Ige TJ, DeLeon P, Nabors LA (2017) Motivational interviewing in an obesity prevention program for children. Health Promot Pract 18: $263-274$.

3. Nabors L, Burbage M, Woodson K, et al. (2015) Implementation of an after-school obesity prevention program: Helping young children toward improved health. Issues Compr Pediatric Nurs 38: 22-38.

4. Nabors L, Welker K, Pavilack B, et al. (2018) Evaluation of an after-school obesity prevention program for children. Int $J$ of Child Nutr and Health 7: 1-8.

5. Sacko RS, Brazendale K, Brian A, et al. (2018) Comparison of indirect calorimetry- and accelerometry- based energy expenditure during object project skill performance. Meas Phys Educ Exerc Sci 1-11.

DOI: $10.36959 / 569 / 454$

Copyright: (C) 2019 Welker K, et al. This is an open-access article distributed under the terms of the Creative Commons Attribution License, which permits unrestricted use, distribution, and reproduction in any medium, provided the original author and source are credited. 